\title{
Research on the mobile library marketing service of university library under the new media environment
}

\author{
Xiuli Li ${ }^{*}$, Chunyue Liu \\ JiLin Business and Technology College, Changchun, China \\ * Corresponding author: Xiuli Li, Master's degree, lixiulibb@126.com
}

\begin{abstract}
With the development of new media technology, mobile library service has become the main mode of University Library service. This paper introduces the concept of mobile library, service model and marketing strategy to improve the service quality of University Library and enhance service effect.
\end{abstract}

Key words: Mobile library; marketing; WeChat; two dimensional code

\section{Introduction}

With the development of wireless mobile network and multimedia technology, mobile users in Colleges and universities are increasing constantly. In the new media environment, more and more attention has been paid to how to develop mobile information service marketing in University libraries. Micro-blog, WeChat and other service model has become the main source of information release and audience communication platform, the continuous development of these service modes will bring more problems to the University library. The author analyzes the service marketing mode of mobile library of University Library under the new media environment at present, the integrated marketing strategy, to improve the modern university library micro marketing service theory, enhance the interaction between readers and improve the research of library information service ability and other aspects of the certain reference.

\section{Concept and connotation of mobile library}

Mobile library is an application system that does not accept time, place and IP address restrictions, and allows readers to use library resources at any time and anywhere through mobile phones, tablet PCs and other mobile devices. With the rapid development of Internet technology and the application of new media technology, the mobile library is given a new meaning ${ }^{1}$. In addition to other mobile devices other than mobile phone including IPad, IPhone is a carrier in considering for library mobile services. These carriers are increasingly involved in people's lives, and have almost dominated the portable communication. The function of mobile library is mainly embodied in carrying out mobile service. After the SMS service, 
WAP website service and APP service stage, the mobile library is more diversified and richer in the form of service. SMS, WAP, APP and other 3 services are not replacing each other, but tend to integrate and integrate their respective service advantages. The greatest advantage of mobile libraries is the ability to provide ubiquitous and ubiquitous library services. The ideal goal of library service is that any reader in any place can obtain any library resources at any time. Without doubt, the mobile library is the ideal mode of Library Service.

\section{Service mode, marketing and application of mobile library}

\subsection{SMS service}

SMS has always been the most intense and truly interesting service for mobile library users. Using SMS to push all kinds of information, it can bring direct help to the users. For example, there are 30 thousand registered mobile phone users in Tsinghua University mobile library, and the use of SMS is about 2000000 of the cumulative use in a few years ${ }^{2}$. SMS service trends have a very obvious upward trend. In addition to the OPAC reservation, mobile phone SMS to use domain information push to the reader in the system, including the study room reservation, qualifying system space management system in advance and so on, is to use SMS as an important way of sending information actively.

\subsection{MMS service}

In terms of increased capacity, MMS has a greater advantage over text messaging. However, due to the restrictions of non technical factors and the high cost of sending MMS, the current MMS service which is pushed by SMS is aimed at a relatively large number of periodicals in the integrated system and In the normal WAP interface, the teacher is allowed to order the journals he cares about, If it provides a push service for RSS's latest article, then the system sends the push message to the registered teacher.

\subsection{WAP mobile web services}

WAP is an internet protocol, for the majority of users to visit the WAP website to provide technical support. Domestic libraries set up special WAP servers for readers ${ }^{3}$, so as to realize the retrieval of various types of library resources, and even make an appointment and renew. The realization of a simple service, the electronic resources to try, the business information content of third parties, including its cover, watercress directory, reviews and evaluation are embedded into the mobile phone library. Slowly developed until now a scholar of mobile WAP, although most of the OPAC library functions are migrating to mobile phone, in addition to this migration, should also do more to develop their own unique function of the mobile phone, but migration is the most basic, but also make up the fastest, most easily effect. In addition, with the development of technology, there are many systems that directly support the mobile version, and the library has integrated it. In fact, some foreign library resources integration includes the so-called mobile library, it is not like our understanding of the 
resource integration of cross platform retrieval, but it itself already has the support of mobile phone library together. The mobile library or go on business system of roads, which is very important reason is the support of the whole thesis, in the beginning in the lack of technical strength, not to support the reading, whether it is to support the mobile library of the scholar or superstar is essential. In addition, some videos can be pushed on the phone.

\subsection{Mobile client application}

Whether iPhone services or Android mobile client development, are dependent on a single package associated with the realization of the same, rather than the previously mentioned SMS, MMS, WAP website simple interaction, Tsinghua University library do the first client application is Android's development status is to get a book above will have a bar code, through the mobile phone camera to take the bar code bar code, the information will be converted into bibliographic information, the bibliographic information is forwarded to the OPAC system of the library, can show whether the book on the shelf or make an appointment ${ }^{4}$. In the scholar mobile library system, there are already client applications for iPhone and Android. The mobile library either function or function in the construction of the construction of resources, but also should pay attention to resources, is some non academic journal content in Android, Ipad, Iphone can be included in the browser can be read.

\section{Marketing strategy of mobile library service}

Readers are the service objects of the library, satisfying readers' demands is the starting point and end result of library developing various service projects. In order to publicize and promote the in-depth mobile library services, so that readers in a higher level of understanding of the products and services of the mobile library, mobile library service marketing borrowed a concept in economics, the purpose is to improve the mobile library service level ${ }^{5}$.

\subsection{Continue to improve the service content of the mobile library}

The mobile library is not only a function to the reader's appreciation, but also the corresponding resources, this resource is for the resources in the library, in recent years the reader needs for scientific and technical information and resources becomes more and more intense, In fact, libraries use such networks to promote services, and more cases are considered in the academic information environment for the search, discovery, dissemination and sharing of academic literature. Of course, in the current environment, entertainment may be more popular in the mobile internet. How do we seek a balance between the dissemination of scholarly information and the more relaxed use of the Internet?

\subsection{Using WeChat to develop mobile library service marketing}

Mobile phones are not only a tool themselves, but some of their software is also a relatively 
independent tool. If you use a wide range of WeChat, you can put some applications on WeChat. In 2013, the Beijing Technology and Business University library opened the WeChat public platform, with the WeChat public platform increasingly perfect function, in addition to static content, there is an interactive search ${ }^{6}$. WeChat public platform can not only retrieve all resources of the library, the realization of online search for books, online books, renew online consulting problems, at the same time, it can also read online mass electronic books, reading is a person's mental development, character shaping, philosophy of life guidance. Beijing Technology and Business University library takes WeChat public platform as the carrier to provide personalized services for teachers and students in the whole hospital.

\subsection{Micro-blog marketing is a more effective means of information service in mobile libraries}

Micro-blog is a social networking platform for sharing short messages via the care mechanism. At present, in a broad sense, the micro-blog mobile marketing of University Libraries means a series of marketing activities, such as branding, activity planning, information service and product publicity, for mobile users through the micro-blog platform. Micro-blog's platform features is a web side plus mobile terminal, including normal micro-blog content publishing and forwarding, App applications, games and so on.The micro-blog marketing platform of university library is based on the websites with high influence, such as Sina, Sohu and so on. At present, most of these sites have been developed, the big views, network share, the influence is strong, many readers will be in between the use of these sites to find naturally or half unconsciously the official micro-blog university library information service content, at any time.

In the positioning mode, the university library can use multimedia advanced technology in micro-blog, through the release of the text, pictures, video and other forms of information to show the information service products, three-dimensional information service through the description on the related content, the potential readers more visually acceptable information. At the same time, through the micro-blog portal of the university library, readers can understand the latest information recommendation and related hot spots of the university library, which makes the library work more transparent and open.

On user behavior, university library according to the attention of fans, fans including age, occupation, grasp the interest of individual feature data, and through communication with the fans to understand the needs of the fans. Combined with the University Library's own information service promotion plan, different information service contents and forms are formulated to meet the needs of different readers, so as to achieve the purpose of micro-blog marketing.

\subsection{Using two-dimensional code to carry out mobile service marketing}

Mobile phone two dimensional code is one of the functions of mobile phone tools, it plays a 
big role in the information transmission of the library. For example, SMS call with a two-dimensional code function, can put through the two-dimensional code retrieval results. You can also use the two-dimensional code in the new job, using two-dimensional code as a clue, in different places to give readers a hint. The premise is that readers are carrying mobile phones. In addition some libraries use two-dimensional code as an information medium, to control the collection of library open, readers access to a book in any environment, get the two-dimensional code to the shelf stacks, let the shelf identify two-dimensional code, and open the corresponding shelves.

\section{Conclusion}

Mobile library is a multi terminal environment, and readers are scattered in different application fields. The service goal of the mobile library is to enable the reader to use any of the library's services in any application environment. Future mobile libraries can help libraries become a better information maker. Because the phone itself can access library information anytime and anywhere, and the best way for readers to experience is to allow readers to participate in the construction of their own mobile library. The development of the mobile library and the construction of the cloud should be well integrated, so as to achieve seamless migration between different terminals.

\section{Acknowledgements}

This paper is one of the achievements of the research project of marketing of University Libraries under the new media environment, sponsored by the JiLin Business And Technology College in 2017 (Project No.: Social Science [S2017] No. 016 ).

\section{References}

1. Wang Jianjuan. Research on mobile library service in Chinese universities. D. Zhengzhou University, 2013.

2. Wang Qian, Zhang Chengyu. Research on user experience of wireless mobile digital library at Tsinghua University, J. Journal of university library. (2010) 36-43.

3. Ye Hua. The mobile library service mode and marketing strategy of Henan, J. Library Journal.(2016)117-119.

4. Zhang Bei, Dou Tianfang, Zhang Chengyu, Liu Fangsheng. Exploring the mobile service development of University Libraries Based on subject knowledge, J. knowledge management forum.(2014)13-18.

5. Niu Shu. University Library SOLOMO WeChat marketing analysis and design, J. Journal of library. (2017) 83-85.

6. URL: http://www.lib.btbu.edu.cn/ (23.5. 2017.) 